\title{
The in Vitro Effect of Vitamin D on the Antibiotic Susceptibility of Carbapenem Resistant Pseudomonas species
}

\author{
Nahla Sahloul*, Nehad Kamel, Samira Shoeib, Noha M. Gohar \\ Department of Medical Microbiology and Immunology, Faculty of Medicine, Cairo University, Cairo, Egypt
}

Key words:
Pseudomonas, carbapenem
resistance, meropenem,
vitamin D, checkerboard
*Corresponding Author:
Nahla Sahloul
Department of Medical
Microbiology and
Immunology, Faculty of
Medicine, Cairo University,
Cairo, Egypt
Tel.: 01006029682
nahlasahlol@yahoo.com

\begin{abstract}
Background: Carbapenem-resistant Pseudomonas infections become a serious problem with limited options of treatment. Many studies support the role of vitamin $D$ in competing different infections by many mechanisms through enhancing host immune defense. Objective: to assess the antimicrobial effect of vitamin D on carbapenemresistant Pseudomonas. Methodology: Fifty carbapenem-resistant Pseudomonas isolates were studied. All isolates were tested for susceptibility to imipenem and meropenem using disk diffusion method. Modified carbapenem inactivation methods were done for carbapenemase and metallo-beta-lactamase detection. Isolates were tested for MIC of meropenem and vitamin $D$ by broth-microdilution and their combination by checkerboard method. Results: Regarding the combination between meropenem and vitamin D; 10 isolates showed decrease in their MIC of meropenem. One isolate showed additive result, 5 isolates showed decrease more than 2 folds, 4 isolates showed 2 folds decrease. Conclusion: Vitamin D acts as an adjuvant to meropenem rather than having a direct antimicrobial effect on carbapenem-resistant Pseudomonas species.
\end{abstract}

\section{INTRODUCTION}

Carbapenems are the most effective antimicrobial agents against Gram-positive and Gram-negative bacteria including Pseudomonas aeruginosa $(P$. aeruginosa). ${ }^{\mathbf{1 , 2}}$ Carbapenem resistance mechanisms have immerged under the pressure of carbapenem use in clinical settings and may be classified as enzymatic, which mediated by carbapenemases and nonenzymatic. Carbapenem resistance however, develops frequently due to the concomitant presence of more than one mechanism. ${ }^{1}$ Infections caused by carbapenemresistant $P$. aeruginosa (CRP) are associated with devastating outcomes. The World Health Organization categorized CRP among the most critical bacterial pathogens that need urgent new alternatives of treatment. $^{3}$ Studies have reported several novel nonantibiotic therapeutic approaches in competing antibiotic-resistant $P$. aeruginosa, these therapeutic approaches can be used as an alternative or in combination with conventional antibiotic treatment. ${ }^{4}$ One of these alternatives is vitamin $\mathrm{D}$, which is thought to have antimicrobial actions through a variety of mechanisms; as it enhances innate immunity, modulates adaptive immunity and may have a direct antimicrobial effect. It has potential antimicrobial actions against different organisms, such as bacteria, viruses and fungi. Vitamin D is inexpensive, and adequate supplementation can be achieved at minimal cost. Vitamin D use could reduce inappropriate antibiotic prescription and boost therapeutic response when combined with appropriate antibiotic use. ${ }^{5}$
The aim of the present study was to assess the antimicrobial modulatory effect of vitamin D alone and its combined effect with meropenem on carbapenem resistant Pseudomonas species and to study the prevalence of carbapenemase production among them.

\section{METHODOLOGY}

This study involved 50 Pseudomonas isolates collected from Kasr Al-Ainy hospitals' laboratories during the period from April 2020 to March 2021.

All isolates were stored in glycerol broth at $-80{ }^{\circ} \mathrm{C}$ prior to the further study.

All isolates were subjected to:

Culture and identification of Pseudomonas colonies:

Each isolate was subcultured on MacConkey's medium (Oxoid, UK) and incubated aerobically at 37 ${ }^{\circ} \mathrm{C}$ for 24 hours. Identification of Pseudomonas at the genus level was confirmed by conventional methods including: colony morphology, Gram-stained smear, biochemical reactions (oxidase tests and triple sugar iron). ${ }^{6}$

Antimicrobial susceptibility testing by disk diffusion method:

All Pseudomonas isolates were tested for their susceptibility to imipenem and meropenem using KirbyBauer disk diffusion method. ${ }^{7}$ Imipenem (IPM 10 $\mu \mathrm{g}$ ) and meropenem (MEM 10 $\mu \mathrm{g}$ ) disks (Himedia, India) were placed on the inoculated Mueller Hinton agar plates (Oxoid, UK), then were incubated aerobically 
overnight at $37^{\circ} \mathrm{C}$. The diameter of each zone of inhibition was measured in $\mathrm{mm}$ and was interpreted using the CLSI breakpoints. ${ }^{\mathbf{2}}$ Pseudomonas isolates which showed zone of inhibition $\leq 15 \mathrm{~mm}$ to imipenem and meropenem were interpreted as carbapenem resistant and enrolled in our study.

Phenotypic detection of carbapenemases production by modified carbapenem inactivation methods $(\mathrm{mCIM}):^{8}$

A meropenem disk was placed in a suspension of tested carbapenem resistant pseudomonas isolate and incubated for a few hours.

- The strain was tested for carbapenemase activity.

- In carbapenemase producer bacterium, the carbapenem in the disk was hydrolyzed by the carbapenemase. When the disk was then transferred to a plate that had just been inoculated with a meropenem-susceptible E. coli and the plate was incubated overnight, there was no zone or a very small zone of inhibition around the meropenem disk.

- In contrast, in non carbapenemase producer bacterium, the meropenem disk retained its activity and was available to inhibit growth of E. coli as evidenced by a zone of inhibition around the disk.

- The isolates that expressed carbapenemase activity were tested for metallo beta-lactamases by EDTA modified carbapenemase inactivation method (eCIM).

- $20 \square 1$ of $0.5 \mathrm{M}$ EDTA were added to another suspension of carbapenemase producing isolate and the previous steps were repeated.

- The zone of inhibition was measured with and without EDTA and interpreted according to CLSI $2021^{8}$

Determination of minimal inhibitory concentrations (MICs) using broth micro dilution method of meropenem alone, vitamin $D$ alone and their combination by checkerboard method:

- Meropenam stock solution: Meropenam (meronam $500 \mathrm{mg}$ vial) (Pfizer, USA) was dissolved in $5 \mathrm{ml}$ sterile distilled water then diluted to get the stock solution of $512 \mu \mathrm{g} / \mathrm{ml}$, which was subsequently used to prepare the varying antimicrobial dilutions. ${ }^{8}$ (The dilutions used were from $128 \mu \mathrm{g} / \mathrm{ml}$ to $0.25 \mu \mathrm{g} / \mathrm{ml})$.

- Vitamin D stock solution: Vitamin D (Devarol S 200000 IU) (Memphis, Egypt) was dissolved in propylene glycol, then diluted in sterile distilled water to get the stock solution of $2048 \mu \mathrm{g} / \mathrm{ml}$, which was subsequently used to prepare the varying dilutions., 10 (The dilutions used were from 512 $\mu \mathrm{g} / \mathrm{ml}$ to $1 \mu \mathrm{g} / \mathrm{ml})$.

- Checkerboard method to test meropenem and vitamin D combination: ${ }^{11}$

- The MIC of each separate agents was determined on different sides of the same plate. Twofold dilutions of the meropenem was placed in rows in ascending concentrations (except the first row). Vitamin D was similarly distributed among the columns (except the first column). The two agents were dispensed into combination panel (50 $\mu \mathrm{l}$ volume of each prepared dilution was distributed in wells, thus $100 \mu \mathrm{l}$ final volume per well, thus, each well contained a unique combination of concentrations of the two drugs. The first row contained only vitamin $\mathrm{D}$, while, the first column contained only the meropenem.

- Growth and sterility controls was included in all plates.

- A bacterial inoculum of $5 \times 10^{5} \mathrm{CFU} / \mathrm{ml}$ was prepared, $100 \mu \mathrm{l}$ volume of each tested isolate was added to wells. Microtiter plates were incubated at $37^{\circ} \mathrm{C}$ for $18-20$ hours.

- The fractional inhibitory concentration index (FICI) of each drug was calculated from the following equation:

○ $\mathrm{FICI}=(\mathrm{MIC}$ of drug $\mathrm{A}$ in combination/ MIC of drug $\mathrm{A}$ alone) +(MIC of drug $\mathrm{B}$ in combination/MIC of drug B alone)

○ Synergy is defined as a FICI score of $\leq 0.5$, Antagonism is defined as a FICI score of $\geq 2$, No interaction (indifference) is a score of $1<$ FICI $<2$, Addition is a score of $1 \leq$ FICI $<0.5$.

\section{Statistical Analysis}

Data were coded and entered using the statistical package for the Social Sciences (SPSS) version 26 (IBM Corp., Armonk, NY, USA). Data was summarized using frequency (count) and relative frequency (percentage) for categorical data. For comparing categorical data, Chi square $\left(\chi^{2}\right)$ test was performed. Exact test was used instead when the expected frequency is less than 5. P-values less than 0.05 were considered as statistically significant. ${ }^{\mathbf{1 2}}$

\section{RESULTS}

The present study was performed on 50 carbapenem resistant Pseudomonas isolates, which were identified by their colony morphology on MacConkey's agar and standard biochemical reactions. Carbapenem resistance of all isolates was confirmed by presence of zone of

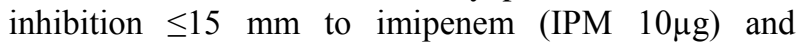
meropenem (MEM 10 $\mu \mathrm{g}$ ) disks using Kirby-Bauer disk diffusion method. 
Phenotypic detection of carbapenemases production by modified carbapenem inactivation methods (mCIM)

In the present study, out of 50 Pseudomonas isolates, 16 isolates $(32 \%)$ showed carbapenemase activity by mCIM, while 34 isolates (68\%) were noncarbapenemase producers. Out of the 16 isolates which were positive for carbapenemase production, 9 (18\%) showed metallo beta-lactamase activity by eCIM (Figure 1).

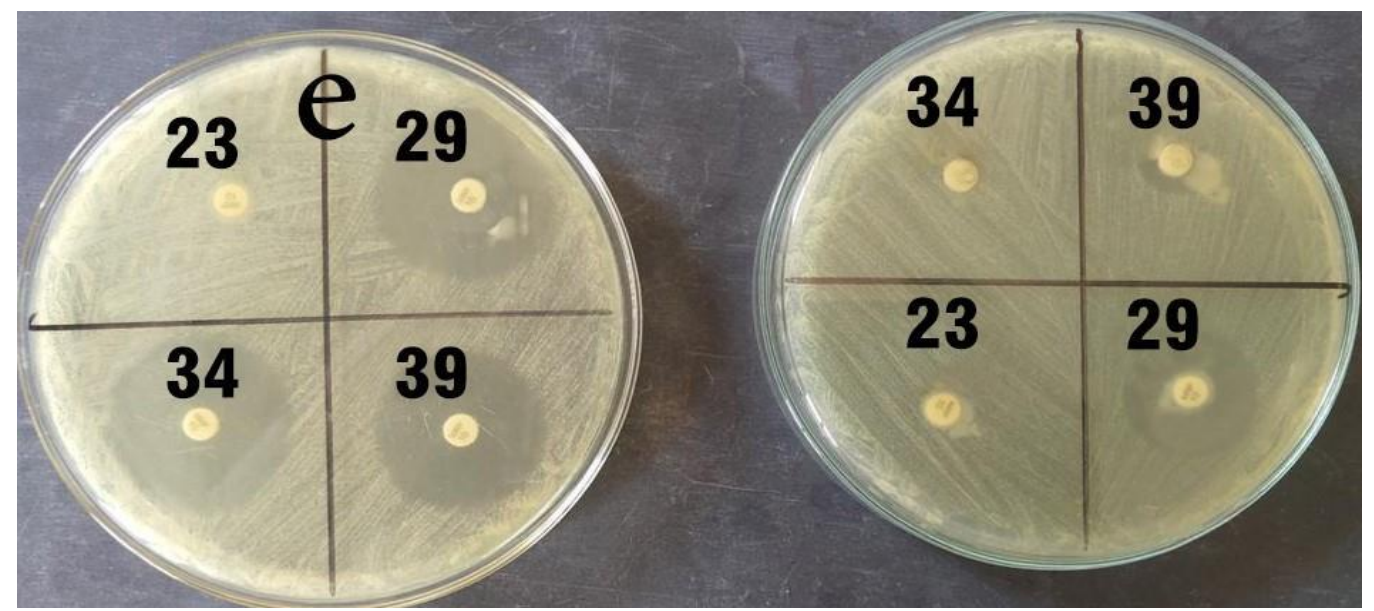

Fig. 1: Detection of carbapenemases production by mCIM (right plate) and eCIM (left plate), isolate no. (29) was a non-carbapenemase producer (zone diameter $\geq 19 \mathrm{~mm}$ ), isolate no. $(23,34,39)$ were carbapenemase producers (zone diameter $\leq 6-15 \mathrm{~mm}$ ), isolates no. (34 and 39) showing metallo beta-lactamase activity (there is increase in zone diameter for eCIM vs. zone diameter of $\mathrm{mCIM} \geq 5 \mathrm{~mm}$ ).

Minimal inhibitory concentration (MIC) of meropenem and vitamin $D$ and their combinations

- Minimal inhibitory concentration of meropenem: Out of 50 Pseudomonas isolates, MIC of meropenem was more than $128 \mu \mathrm{g} / \mathrm{ml}$ in 42 isolates $(84 \%)$, while in 8 isolates (6\%) MIC was $128 \mu \mathrm{g} / \mathrm{ml}$ or less.

- Minimal inhibitory concentration of vitamin D: Out of 50 Pseudomonas isolates, only one isolate (2\%) showed MIC of vitamin D $8 \mu \mathrm{g} / \mathrm{ml}$, while in 49 isolates (98\%) MIC was more than $512 \mu \mathrm{g} / \mathrm{ml}$.

- Minimal inhibitory concentration of both meropenem and vitamin $D$ :

The combined effect of meropenem and vitamin D was tested by using Checkerboard method. The results were interpreted by reporting the decrease in folds of meropenem MIC with addition of vitamin D. The FIC index was calculated (if possible) and was interpreted.

Out of 50 Pseudomonas isolates, 10 isolates (20\%) showed decrease in MIC of meropenem (increase sensitivity to meropenem) with addition of vitamin
D. While 40 isolates $(80 \%)$ showed no effect with addition of vitamin D. Out of 10 isolates which showed decrease in MIC of meropenem with addition of vitamin D; Additive result (FICI $=0.53$ ) was observed in one isolate $(2 \%)$, while in the other 9 isolates, FICI could not be calculated as this equation needs to determine MIC of each drug alone, which was not possible as vitamin D MIC was not determined ( $>512 \mu \mathrm{g} / \mathrm{ml}$ ).

The results of nine isolates could be interpreted according to decrease in MIC of meropenem (2 folds or more than 2 folds decrease in MIC). Therefore, 5 isolates (10\%) showed a decrease in MIC of meropenem more than 2 folds when meropenem combined with vitamin D (Figure 2), while 4 isolates $(8 \%)$ showed 2 folds decrease in meropenem MIC when combined with vitamin D. There was a significant relation between addition of vitamin D and increasing sensitivity of carbapenem resistant pseudomonas to meropenem ( $p$-value $=0.012$ ). (Table 1) 
Table 1: MIC of meropenem, vitamin D and their combination:

\begin{tabular}{|c|c|c|c|}
\hline \multirow{2}{*}{ Meropenem } & MIC & Count & \% \\
\hline \multirow{2}{*}{ Vitamin D } & $\mathbf{1 2 8}$ & 42 & $84 \%$ \\
\hline \multirow{2}{*}{$\begin{array}{c}\text { Combined effect of } \\
\text { vitamin D and meropenem }\end{array}$} & $\mathbf{1 1 2 8}$ & 8 & $16 \%$ \\
\cline { 2 - 4 } & decrease in meropenem MIC> 2folds & 49 & $98 \%$ \\
\hline & decrease in meropenem MIC 2folds & 4 & $2 \%$ \\
\cline { 2 - 4 } & no effect & 40 & $20 \%$ \\
\hline
\end{tabular}

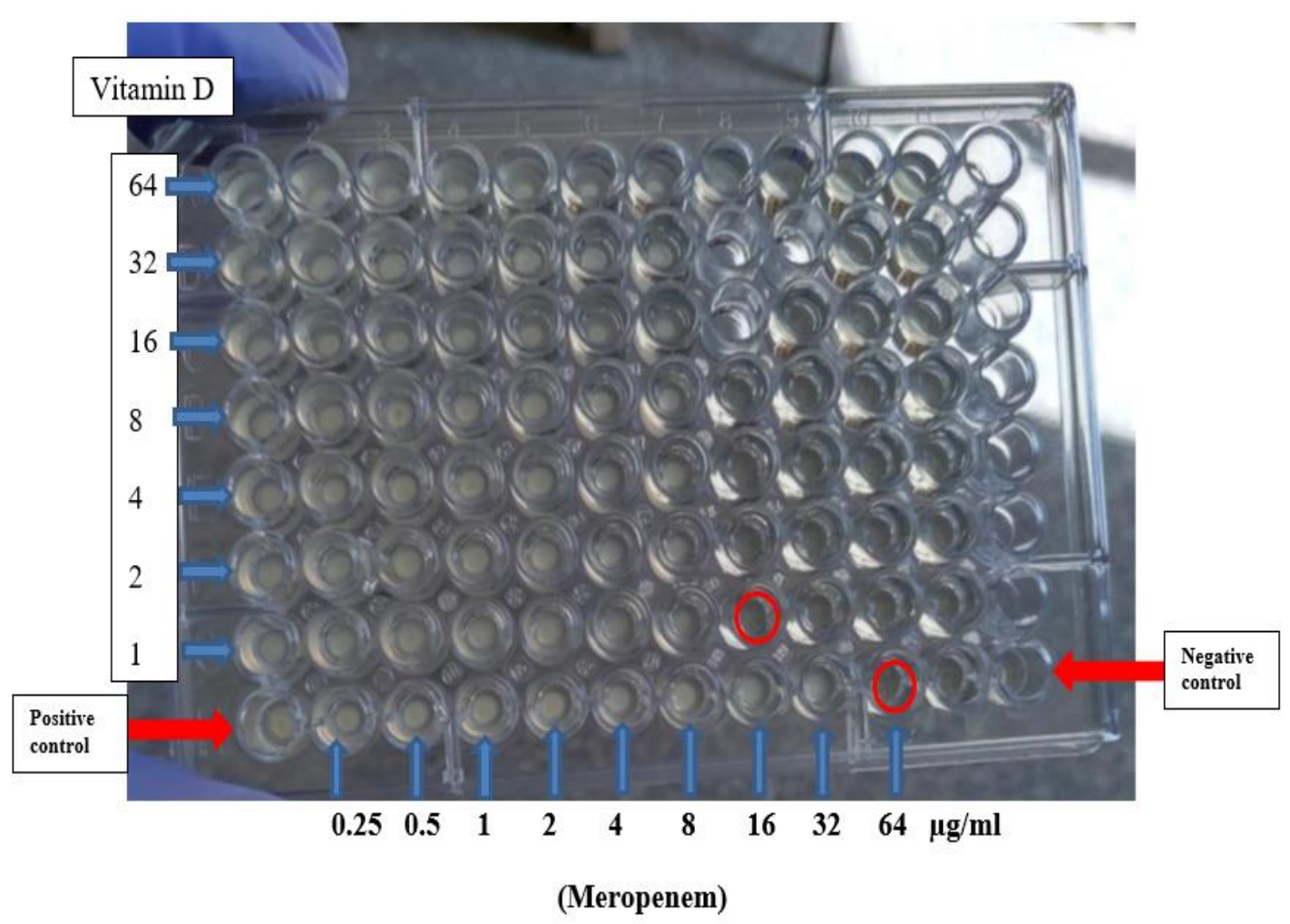

Fig. 2: Checkerboard method of Pseudomonas isolate showing 4 folds decrease in MIC of meropenem. Meropenem MIC was $64 \mu \mathrm{g} / \mathrm{ml}$ while vitamin D MIC was not determined and when combined with $1 \mu \mathrm{g}$ of vitamin D, meropenem MIC became $16 \mu \mathrm{g} / \mathrm{ml}$.

\section{DISCUSSION}

Carbapenem resistance exhibited by $P$. aeruginosa occurs through many mechanisms; either secreting carbapenemase enzyme, decreasing membrane permeability or through efflux pumps, also formation of biofilm can play a role in resistance. ${ }^{13}$

Vitamin D is considered as a hormone that has many actions other than calcium level regulation. It was used in the pre-antibiotic era in treating tuberculosis. ${ }^{14}$ There are many studies support its role in competing different types of infections in vivo by many mechanisms through enhancing host immune defense. ${ }^{5}$ Other studies suggest that it also may have a direct antibacterial action by itself, the suggested mechanisms are still not well understood, but many of them suggest that it may alter the membrane permeability of bacteria, so may help the entrance and the action of antibiotics. ${ }^{15,16,17}$ The current study was designed to assess the antimicrobial modulatory effect of vitamin D alone and its combined effect with meropenem on 50 carbapenem resistant Pseudomonas isolates and to study the prevalence of carbapenemase production among them.

In the present study, the 50 carbapenem resistant Pseudomonas isolates were classified by modified carbapenem inactivation methods (mCIM) to carbapenemases and non-carbapenemase producers. Carbapenemase producing Pseudomonas isolates were 16 isolates $(32 \%)$. Similar results were obtained by other studies as which reported that $28 \%$ and $33 \%$ of the 
Pseudomonas isolates were carbapenemase producers, respectively. ${ }^{\mathbf{1 8 , 1 9}}$ Higher rates were detected by many studies which reported that $75.4 \%$ and $62.74 \%$ of Pseudomonas isolates were carbapenemase producers respectively. ${ }^{\mathbf{2 0}, \mathbf{2 1}}$ Lower rates were detected by other studies which reported that $18.7 \%$ and $21.36 \%$ of Pseudomonas isolates were carbapenemase producers respectively. $^{22,23}$

The differences in the percentage of carbapenamase production could be attributed to the presence of different types of carbapenemases that also transferred by mobile genetic elements. Therefore, its prevalence is closely depending on geographic region, methods of testing and the major variability in application of infection control guidelines and antibiotic stewardships, which affect spread of resistant strains. ${ }^{\mathbf{2 4}}$

Minimum inhibitory concentration of meropenem in $84 \%$ of the isolates was more than $128 \mu \mathrm{g} / \mathrm{ml}$ in this study; reflecting high resistance in these isolates. This finding was in accordance with a study which revealed high level of meropenem resistance (MIC > $64 \mu \mathrm{g} / \mathrm{ml}$ ), which was allied to multiple mechanisms including carbapenemase production and efflux-pump overexpression. $^{25}$ Another study showed different levels of meropenem resistance among carbapenem resistant Pseudomonas isolates. Most of them exhibited high level of meropenem resistance (MIC was from 64 to $128 \mu \mathrm{g} / \mathrm{ml}$ ), while others showed intermediate level of meropenem resistance (MIC was from 16 to 32 $\mu \mathrm{g} / \mathrm{ml}){ }^{\mathbf{2 6}}$ Lower levels of MIC to meropenem among carbapenem resistant $P$. aeruginosa isolates were detected (MIC was $8 \mu \mathrm{g} / \mathrm{ml}$ and $16 \mu \mathrm{g} / \mathrm{ml}$ ) as these isolates appear to express only efflux pump genes. ${ }^{27}$ The high level of meropenem resistance in different studies can be explained by overlapping of carbapenem resistance mechanisms. Many studies attributed the high level of meropenem resistance to loss or mutation of outer membrane porin D (Orp D), carbapenemase production especially metallo beta-lactamases and high expression of efflux pumps. ${ }^{\mathbf{2 5 , 2 6}}$

Minimum inhibitory concentration of vitamin D for 49 isolates $(98 \%)$ was $>512 \mu \mathrm{g} / \mathrm{ml}$ in this study. Another study revealed that the MIC of vitamin D was > $1024 \mu \mathrm{g} / \mathrm{ml}$ indicating that vitamin D did not exhibit clinically relevant antibacterial activity. ${ }^{9}$

In our study, the MIC of vitamin D of only one isolate was $8 \mu \mathrm{g} / \mathrm{ml}$, this was in agreement with a study which tested the effect of vitamin D on Streptococcus mutans, where the MIC of vitamin D for three isolates was $16 \mu \mathrm{g} / \mathrm{ml}$, and $8 \mu \mathrm{g} / \mathrm{ml}$ for one isolate. ${ }^{15}$ Similarly, MIC of vitamin D against Porphyromonas gingivalis ranged from 3.125 to $6.25 \mu \mathrm{g} / \mathrm{ml}$ in another study. ${ }^{28}$

The decreased in vitro activity of vitamin $\mathrm{D}$ on bacteria can be attributed firstly to the concept that vitamin $\mathrm{D}$ act as an adjuvant rather than having direct antimicrobial properties, as it acts only through changing permeability of bacterial membrane to antibiotic by mechanisms that still not clear. ${ }^{9,16}$ Secondly, it may act through mechanisms mimics its action on human cell through changing levels of different ions in the cell (calcium and zinc) that may need specific environment to show these actions. ${ }^{29,30}$

In the current study, addition of vitamin D to meropenem resulted in decreased MIC of meropenem in $20 \%(10 / 50)$ of isolates. There was a significant relation ( $p$-value $=0.012$ ) between addition of vitamin $\mathrm{D}$ and increasing sensitivity of carbapenem resistant Pseudomonas to meropenem.

Another study tested the effect of vitamin D on increasing sensitivity of Pseudomonas to amikacin and other antibiotics, it was found that vitamin $\mathrm{D}$ had no direct action when used alone but improved sensitivity of MDR Pseudomonas isolate to gentamicin and amikacin, this could be attributed to increase membrane permeability caused by vitamin $D$ facilitating the influx of amikacin and gentamicin. ${ }^{\mathbf{1 6}}$ Other studies supported the antimicrobial effect of vitamin D on Streptococcus mutans, ${ }^{\mathbf{1 5}}$ Porphyromonas gingivalis, $^{28}$ and Helicobacter pylori. ${ }^{31}$

In our study, it was found that out of the 10 isolates that showed a decrease in MIC of meropenem on addition of vitamin D, 5 (10\%) of them showed more than 2 folds decrease. Another study showed only one MDR Pseudomonas isolate that showed a decrease in MIC of aminoglycosides more than 2 folds when vitamin $\mathrm{D}$ was added. ${ }^{\mathbf{1 6}}$

It was observed that $8 \%(4 / 10)$ of the isolates in our study showed 2 folds decrease in MIC of meropenem on addition of vitamin D. Similar results were obtained by another study which detected 2 folds decrease in MIC of bacitracin when combined with vitamin $\mathrm{D}$ on Streptococcus sanguinis $(32 \rightarrow 16 \mu \mathrm{g} / \mathrm{ml})$, Lactococcus lactis $(4 \rightarrow 2 \mu \mathrm{g} / \mathrm{ml})$ and Staphylococcus aureus $(128 \rightarrow$ $64 \mu \mathrm{g} / \mathrm{ml}){ }^{15}$

In the present study, FICI that could be calculated in one isolate (2\%) was 0.53 indicating additive effect. Additive effect was also obtained when the effect of vitamin D was studied on Porphyromonas gingivalis and combined with metronidazole $($ FICI $=0.75)$ and when combined with tetracycline $($ FICI $=1.0) .{ }^{\mathbf{2 8}}$

Several studies have demonstrated the direct antibacterial activity of vitamin D against both Grampositive and Gram-negative bacteria, its mechanism of action might be through increasing permeability of bacteria to antibiotics. Lipid-soluble compounds including vitamin $\mathrm{D}$ have been found to cause changes in the fluidity of the bacterial membrane, facilitating the penetration of synthetic substances, including antibiotics. ${ }^{16,32}$ Vitamin D might act by inhibiting bacterial efflux systems. ${ }^{\mathbf{9 , 1 5}}$ Another study proposed a possible mechanism for the antibacterial action of vitamin D. Vitamin D attached to the bacterial cell wall through interactions with the peptidoglycan of Streptococcus sobrinus and Streptococcus mutans. Its 
adherence to the cell wall caused disruption to the bacterial cell wall and membrane, making them shrink, become rough and increase the internal cellular pressure causing bleb-like formation and eventually causing cell membrane rupture and bacteria damage. Based on scanning electron microscopy findings, it is evident that vitamin D3 is considered a membrane-active agent and is toxic to these oral bacteria. ${ }^{33}$

Another possible mechanism of action of vitamin D through affecting the zinc level inside the bacterial cell. Zinc is an essential element involved in the expression of metallo $\beta$-lactamases and in downregulation of Orp D in $P$. aeruginosa. Conversely, zinc can be toxic to bacteria when present in excess, so intracellular levels must be tightly controlled, therefore affecting the zinc level in bacteria (by decreasing or increasing), increasing susceptibility of Pseudomonas to carbapenems. ${ }^{34,35}$ Another study revealed that calcium phosphate inorganic nanoparticles have an inhibitory effect on many bacteria including Pseudomonas, they also found restoration of the activity of many resistant antibiotics. Based on this study, vitamin D may exert its action through affecting the calcium metabolism of the bacteria in a way similar to its action on human cells. 29,36

The action of vitamin D directly on microbes is still an obscure issue. Further in vitro studies are needed to detect the mechanism of interaction between antibiotics and vitamin $\mathrm{D}$, as well as, its interaction with bacterial cells; whether the bacteria express vitamin D receptors or not and the spectrum of activity of vitamin D.

\section{CONCLUSION}

Vitamin D acts as an adjuvant to meropenem rather than having a direct antimicrobial effect on carbapenem resistant Pseudomonas species. Further studies are needed on larger numbers of Pseudomonas isolates and other problematic Gram-negative and Gram-positive bacteria with wider selection of the antibiotics under different in vitro environmental conditions to detect the mechanism of interaction between antibiotics and vitamin $\mathrm{D}$ as well as their interaction with bacterial cells.

This manuscript has not been previously published and is not under consideration in the same or substantially similar form in any other reviewed media. I have contributed sufficiently to the project to be included as author. To the best of my knowledge, no conflict of interest, financial or others exist. All authors have participated in the concept and design, analysis, and interpretation of data, drafting and revising of the manuscript, and that they have approved the manuscript as submitted.

\section{REFERENCES}

1. Meletis G, Exindari M, Vavatsi N, et al. Mechanisms responsible for the emergence of carbapenem resistance in Pseudomonas aeruginosa. Hippokratia; 2012; 16(4):303-307.

2. Codjoe F and Donkor E. Carbapenem Resistance: A Review. Med Sci (Basel).; 2017; 6(1):1.

3. Tacconelli E, Carrara E, Savoldi A, et al. Discovery, research, and development of new antibiotics: the WHO priority list of antibioticresistant bacteria and tuberculosis. Lancet Infect Dis.; 2018;18(3):318-327.

4. Pang Z, Raudonis R, Glick B, et al. Antibiotic resistance in Pseudomonas aeruginosa: mechanisms and alternative therapeutic strategies. Biotechnol Adv.; 2019; 37(1):177-192.

5. Youssef D, Miller C, El-Abbassi A, et al. Antimicrobial implications of vitamin D. Dermatoendocrinol.; 2011; 3(4):220-229.

6. UK Standards for Microbiology Investigations. Identification of Pseudomonas species and other Non-Glucose Fermenters. Bacteriology Identification; ID. 2015; 17(3):1-41.

7. Cheesbrough M. Microbiological tests. In: Direct Laboratory Practice in TropicalCountries, Part 2, 2nd edition. Cambridge low price edition 2007. Cambridge University Press: 1-266.

8. Clinical and Laboratory Standard Institute (CLSI, 2021): Performance standards for antimicrobial susceptibility testing; 28th informational supplement. M 100-S-28. Wayne, PA, USA, 2021.

9. Tintino S, Morais-Tintino C, Campina F, et al. Action of cholecalciferol and alpha-tocopherol on Staphylococcus aureus efflux pumps. EXCLI J.; 2016; 15:315-322.

10. Glowka E, Stasiak J, Lulek J. Drug delivery systems for Vitamin D Supplementation and Therapy. Pharmaceutics; 2019; 11(7):347.

11. Leber A. Synergism Testing: Broth Microdilution Checkerboard and Broth Macrodilution Methods (in Isenberg HD ed), In Clinical Microbiology Procedures Handbook (4 ${ }^{\text {th }}$ Edition); 2016; Ch:5.16: p 1-23, ASM Press, Washington, DC.

12. Chan Y. Biostatistics 103: Qualitative Data-Tests of Independence. Singapore Med J. 2003, 44(10): 498-503.

13. McGowan J. Resistance in nonfermenting Gram negative bacteria: multidrug resistance to the maximum. Am J Med.; 119(6 Suppl 1): 2006, S2936; discussion S62-70.

14. DeLuca H. Vitamin D: Historical Overview. Vitam Horm.; 2016. 100:1-20.

15. Saputo S, Faustoferri R, Quivey R. Vitamin D compounds are bactericidal against Streptococcus mutans and target the bacitracin-associated efflux 
system. Antimicrob Agents Chemother.; 2017; 62(1):e01675-17.

16. Andrade J, Morais Braga M, Guedes G, et al. Cholecalciferol, Ergosterol, and Cholesterol enhance the antibiotic activity of drugs. Int J Vitam Nutr Res.; 2018; 88(5-6):244-250.

17. Golpour A, Bereswill S, Heimesaat $M$. Antimicrobial and immune-modulatory effects of vitamin D provide promising antibioticsindependent approaches to tackle bacterial infections - Lessons Learnt from a Literature Survey. Eur J Microbiol Immunol. (Bp).; 2019; 9(3):80-87.

18. Liakopoulos A, Mavroidi A, Katsifas E, et al. Carbapenemase-producing Pseudomonas aeruginosa from central Greece: molecular epidemiology and genetic analysis of class I integrons. BMC Infect Dis.; 2013; 13:505.

19. Lisboa L, Turnbull L, Boyd D, et al. Evaluation of a Modified Carbapenem Inactivation Method for Detection of Carbapenemases in Pseudomonas aeruginosa. J Clin Microbiol.; 2017; 56(1):e0123417.

20. Saavedra S, Duarte C, González M, et al. Characterization of isolates of carbapenemaseproducing Pseudomonas aeruginosa from seven Colombian provinces. Biomedica.; 2014; 34 (Suppl 1):217-223

21. Verma N, Prahraj A, Mishra B, et al. Detection of carbapenemase-producing

Pseudomonas aeruginosa by phenotypic and genotypic methods in a tertiary care hospital of East India. J Lab Physicians; 2019; 11(4):287-291.

22. Lin K, Lauderdale T, Wang J, et al. Carbapenemresistant Pseudomonas aeruginosa in Taiwan: Prevalence, risk factors, and impact on outcome of infections. J Microbiol Immunol Infect.; 2016; 49(1):52-59.

23. Kindu $M$, Derseh L, Gelaw B, et al. Carbapenemase-Producing Non-GlucoseFermenting Gram-Negative Bacilli in Africa, Pseudomonas aeruginosa and Acinetobacter baumannii: A Systematic Review and MetaAnalysis. Int J Microbiol.; 2020; 9461901.

24. Yoon E and Jeong S. Mobile Carbapenemase Genes in Pseudomonas aeruginosa. Front Microbiol.; 2021; 12:614058.

25. Hassuna N, Darwish M, Sayed M, et al. Molecular epidemiology and mechanisms of high-level resistance to Meropenem and Imipenem in
Pseudomonas aeruginosa. Infect Drug Resist.; 2020; 13:285-293.

26. Chalhoub H, Sáenz Y, Rodriguez-Villalobos H, et al. High-level resistance to meropenem in clinical isolates of Pseudomonas aeruginosa in the absence of carbapenemases: role of active efflux and porin alterations. Int $\mathrm{J}$ Antimicrob Agents; 2016; 48(6):740-743.

27. Giske C, Borén C, Wretlind B, et al. Meropenem susceptibility breakpoint for Pseudomonas aeruginosa strains hyperproducing mexB mRNA. Clin Microbiol Infect.; 2005; 11(8):662-669.

28. Grenier D, Morin M, Fournier-Larente J, et al. Vitamin D inhibits the growth of and virulence factor gene expression by Porphyromonas gingivalis and blocks activation of the nuclear factor kappa B transcription factor in monocytes. J Periodontal Res.; 2016; 51(3):359-365.

29. Wu V, Tang S, Uskoković V. Calcium Phosphate Nanoparticles as Intrinsic Inorganic Antimicrobials: The Antibacterial Effect. ACS Appl Mater Interfaces; 2018; 10(40):34013-34028.

30. King M, Kayastha B, Franklin M, et al. Calcium Regulation of Bacterial Virulence. Adv Exp Med Biol.; 2020; 1131:827-855.

31. Hosoda K, Shimomura H, Wanibuchi K, et al. Identification and characterization of a vitamin $D_{3}$ decomposition product bactericidal against Helicobacter pylori. Sci Rep.; 2015; 5:8860.

32. Pretto J, Cechinel-Filho V, Noldin V, et al. Antimicrobial activity of fractions and compounds from Calophyllumbrasiliense (Clusiaceae/Guttiferae). Z Naturforsch C J Biosci.; 2004; 59(9-10):657-662.

33. Almoudi M, Hussein A, Abu Hassan M, et al.The antibacterial effects of vitamin D3 against mutans streptococci: an in vitro study. Eur Oral Res.; 2021; 55(1):8-15.

34. Pederick V, Eijkelkamp B, Begg S, et al. ZnuA and zinc homeostasis in Pseudomonas aeruginosa. Sci Rep.; 2015; 5:13139.

35. Velasco E, Wang S, Sanet M, et al. A new role for Zinc limitation in bacterial pathogenicity: modulation of $\alpha$-hemolysin from uropathogenic Escherichia coli. Sci Rep.; 2018; 8(1):6535.

36. Morvová M , Lajdová I, Spustová V, et al. The effect of vitamin $D_{3}$ supplementation on intracellular calcium and plasma membrane calcium ATPase activity in early stages of chronic kidney disease. Physiol Res.; 2014; 63(Suppl 4):S593-S599. 